\title{
Positive Solutions to $n$-Order Fractional Differential Equation with Parameter
}

\author{
Jing-jing Tan $\mathbb{D}^{1},{ }^{1}$ Cong Tan, ${ }^{2}$ and Xueling Zhou ${ }^{3}$ \\ ${ }^{1}$ School of Mathematics and Information Science, Weifang University, Shandong 261061, China \\ ${ }^{2}$ Shenyang Ligong University, Liaoning 110159, China \\ ${ }^{3}$ College of Mathematics and Systems Science, Shandong University of Science and Technology, Qingdao Shandong 266590, China \\ Correspondence should be addressed to Jing-jing Tan; tanjingjing1108@163.com
}

Received 22 February 2018; Accepted 7 May 2018; Published 3 June 2018

Academic Editor: Gennaro Infante

Copyright (C) 2018 Jing-jing Tan et al. This is an open access article distributed under the Creative Commons Attribution License, which permits unrestricted use, distribution, and reproduction in any medium, provided the original work is properly cited.

By employing the properties of the sum operators, we investigate the solutions of the $n$-order fractional differential equations multiple point boundary value problem (in short BVP) with the boundary conditions contains a parameter. We not only obtain the existence and uniqueness of solutions about this BVP but also construct an iterative scheme to approximate the solution which is important for practical application. An example is given to demonstrate the validity of our main results.

\section{Introduction}

In this paper, we focus on the $n$-order fractional differential equations of multiple point BVP:

$$
\begin{aligned}
& -D_{0+}^{\alpha} x(t)=f_{1}(t, x(t), x(t))+f_{2}(t, x(t)), \\
& \quad 0<t<1, n-1<\alpha \leq n, n \geq 2, \\
& x(0)=x^{\prime}(0)=\cdots=x^{(k)}(0)=0, \quad 0 \leq k \leq n-2, \\
& x(1)=\sum_{i=1}^{m-2} \alpha_{i} x\left(\xi_{i}\right)+\lambda, \quad m \geq 3,
\end{aligned}
$$

where $D_{0+}^{\alpha}$ is the $\alpha$-order fractional derivative, $f_{1}:[0,1] \times$ $[0,+\infty) \times[0,+\infty) \rightarrow[0,+\infty)$ and $f_{2}:[0,1] \times[0,+\infty) \rightarrow$ $[0,+\infty)$ are continuous, $\alpha_{i}>0(i=1,2, \ldots, m-2), 0<\xi_{1}<$ $\xi_{2}<\cdots<\xi_{m-2}<1, \sum_{i=1}^{m-2} \alpha_{i} \xi_{i}^{\alpha-1}<1, \lambda$ is a parameter.

Boundary value problem is an important branch of differential equation. In recent years, as a mathematical model, BVP can be processed in many engineering and scientific models. Fractional differential equations BVP has become a hot issue; see the monographs of Lakshmikantham and Vatsala [1], Rudin [2], Samko et al. [3], Agarwal et al. [4, 5], and Webb and Zima [6]. Many excellent results have been reported; see [7-23]. In [24], El-Sayed and Bin-Taher study the following $\mathrm{m}$-point $\mathrm{BVP}$ :

$$
\begin{aligned}
-x^{\prime \prime}(t) & =f\left(t, D_{0+}^{\alpha} x(t)\right), \quad 0<t<1,0<\alpha<1, \\
x(0) & =0, \\
x(1) & =\sum_{i=1}^{m-2} \alpha_{i} x\left(\xi_{i}\right),
\end{aligned}
$$

By using the technical of Kolmogorov compactness criterion, they obtained the results of existence at least of one positive solution. In [25], Zhang studied the following $n$-order BVP:

$$
\begin{array}{r}
-D_{0+}^{\alpha} x(t)=q(t) f\left(t, x(t), \ldots, x^{(n-2)}(t)\right), \\
0<t<1, \alpha \in(n-1, n], n \geq 2, \\
x(0)=x^{\prime}(0)=\cdots=x^{(n-2)}(0)=0, \\
x^{(n-2)}(0)=1 .
\end{array}
$$

The authors obtained the existence of positive solutions. However, many aspects of the theory to the solutions of the 
fractional differential equations BVP need to be explored, such as the solutions of high-order multipoint BVP.

Motivated by the work mentioned above, we focus on the existence and uniqueness of positive solutions for the nonlocal BVP (1) by the technical of a fixed point theorem of a sum operator. In [12], we study the fractional equations with two and three point boundary conditions, where $\alpha \epsilon$ $(1,2)$. In [20], we study the fractional equations of with integral boundary conditions. The new features of this paper are as follows. Firstly, the order of the fractional differential equation is high order with $\alpha \in(n-1, n]$, and the boundary condition is multipoint boundary condition. Compared with [12], the BVP is more general; secondly, the boundary conditions are dependent on a parameter $\lambda$ which is different from paper [20], so the properties of Green function and the construct of the operator are differential. The Green function and the operator are the key to the two BVP.

The paper is organized as follows. In Section 2, we recall some definitions and lemmas. In Section 3, the existence of positive solutions to BVP (1) is obtained. Finally, in Section 4, an illustrative example is also presented.

\section{Preliminaries}

We first recall four classes operator.

The basic space used in this paper is the space $C[0,1]$. Set

$$
P=\{x \in C[0,1] \mid x(t) \geq 0, t \in[0,1]\} .
$$

Let $D=P$ or $D={ }_{P}^{o}$ and $r$ be a real number with $0 \leq r<$ 1. An operator $T: P \rightarrow P$ is said to be

(1) positive homogeneous if it satisfies

$$
T(t x)=t T x, \quad \text { for any } t \in(0,1), x \in P
$$

(2) subhomogeneous if it satisfies

$$
T(t x) \geq t T x, \quad \text { for any } t \in(0,1), x \in P \text {; }
$$

(3) $r$-concave if it satisfies

$$
T(t x) \geq t^{r} T x, \quad \text { for any } t \in(0,1), x \in D ;
$$

(4) $T: P \times P \rightarrow P$ is said to be a mixed monotone operator if it satisfies $T\left(x_{1}, y_{1}\right) \leq T\left(x_{2}, y_{2}\right)$ for any $x_{i}, y_{i}(i=$ $1,2) \in P, x_{1} \leq x_{2}, y_{1} \geq y_{2}$.

Lemma 1 (see [26]). Let $h>\theta$ and $\beta \in(0,1) . T: P \times P \rightarrow P$ is a mixed monotone operator; $S: P \rightarrow P$ is an increasing subhomogeneous operator for any $x, y \in P$, and there exists a constant $\delta_{0}>0$ such that $T(x, y) \geq \delta_{0} S x$. Assume that

(i) for any $t \in(0,1), x, y \in P$,

$$
T\left(t x, t^{-1} y\right) \geq t^{\beta} T(x, y),
$$

(ii) there exists a constant $h_{0} \in P_{h}$ such that $T\left(h_{0}, h_{0}\right) \in P_{h}$ and $\mathrm{Sh}_{0} \in P_{h}$
Then, the operator equation $T(x, x)+S x=x$ has a unique solution $x^{*}$ in $P_{h}$ and for any initial values $x_{0}, y_{0} \in P_{h}$, constructing successively sequences $x_{n}=T\left(x_{n-1}, y_{n-1}\right)+S x_{n-1}$, $y_{n}=T\left(y_{n-1}, x_{n-1}\right)+S y_{n-1}, n=1,2, \ldots$, we have $x_{n} \rightarrow x^{*}$ and $y_{n} \rightarrow x^{*}$ as $n \rightarrow \infty$.

Lemma 2. Given $\in C(0,1) \cap L(0,1)$, the unique solution of

$$
\begin{aligned}
-D_{0+}^{\alpha} x(t) & =f(t), \quad 0<t<1, \\
x(0) & =x^{\prime}(0)=\cdots=x^{(k)}(0)=0, \\
x(1) & =\sum_{i=1}^{m-2} \alpha_{i} x\left(\xi_{i}\right)+\lambda
\end{aligned}
$$

is

$$
x(t)=\int_{0}^{1} G(t, s) f(s) d s+\lambda \frac{t^{\alpha-1}}{1-\sum_{i=1}^{m-2} \alpha_{i} \xi_{i}^{\alpha-1}},
$$

where $n-1<\alpha \leq n, n \geq 2, m \geq 3,0 \leq k \leq n-2$,

$$
G(t, s)=H(t, s)+\frac{t^{\alpha-1}}{1-\sum_{i=1}^{m-2} \alpha_{i} \xi_{i}^{\alpha-1}} \sum_{i=1}^{m-2} \alpha_{i} H\left(\xi_{i}, s\right)
$$

$H(t, s)$

$$
= \begin{cases}\frac{[t(1-s)]^{\alpha-1}-(t-s)^{\alpha-1}}{\Gamma(\alpha)}, & 0 \leq s \leq t \leq 1, \\ \frac{[t(1-s)]^{\alpha-1}}{\Gamma(\alpha)}, & 0 \leq t \leq s \leq 1 .\end{cases}
$$

Proof. By using the properties of the Riemann-Liouville fractional derivative, we can easily get the conclusion, so we omit.

In the follow, we verify the properties of $G(t, s)$.

Lemma 3. For all $t, s \in(0,1), G(t, s)$ is continuous on $[0,1] \times$ $[0,1]$ and

$$
\begin{aligned}
& \frac{t^{\alpha-1}}{1-\sum_{i=1}^{m-2} \alpha_{i} \xi_{i}^{\alpha-1}} \sum_{i=1}^{m-2} \alpha_{i} H\left(\xi_{i}, s\right) \leq G(t, s) \\
& \leq t^{\alpha-1}\left[1+\frac{1}{1-\sum_{i=1}^{m-2} \alpha_{i} \xi_{i}^{\alpha-1}} \sum_{i=1}^{m-2} \alpha_{i} H\left(\xi_{i}, s\right)\right] .
\end{aligned}
$$

Proof. Form the definition of $G(t, s)$, we can get $G(t, s)$ is continuous. Now, we need only to prove that (12) is satisfied. For all $t, s \in(0,1)$, we have

$$
\begin{aligned}
G(t, s) & =H(t, s)+\frac{t^{\alpha-1}}{1-\sum_{i=1}^{m-2} \alpha_{i} \xi_{i}^{\alpha-1}} \sum_{i=1}^{m-2} \alpha_{i} H\left(\xi_{i}, s\right) \\
& \leq[t(1-s)]^{\alpha-1}
\end{aligned}
$$




$$
\begin{aligned}
& +\frac{t^{\alpha-1}}{1-\sum_{i=1}^{m-2} \alpha_{i} \xi_{i}^{\alpha-1}} \sum_{i=1}^{m-2} \alpha_{i} H\left(\xi_{i}, s\right) \\
= & t^{\alpha-1}\left[1+\frac{1}{1-\sum_{i=1}^{m-2} \alpha_{i} \xi_{i}^{\alpha-1}} \sum_{i=1}^{m-2} \alpha_{i} H\left(\xi_{i}, s\right)\right], \\
G(t, s)= & H(t, s)+\frac{t^{\alpha-1}}{1-\sum_{i=1}^{m-2} \alpha_{i} \xi_{i}^{\alpha-1}} \sum_{i=1}^{m-2} \alpha_{i} H\left(\xi_{i}, s\right) \\
\geq & \frac{t^{\alpha-1}}{1-\sum_{i=1}^{m-2} \alpha_{i} \xi_{i}^{\alpha-1}} \sum_{i=1}^{m-2} \alpha_{i} H\left(\xi_{i}, s\right) \\
= & \frac{1}{1-\sum_{i=1}^{m-2} \alpha_{i} \xi_{i}^{\alpha-1}} t^{\alpha-1} \sum_{i=1}^{m-2} \alpha_{i} H\left(\xi_{i}, s\right)
\end{aligned}
$$

Hence (3) is held; the proof is complete.

\section{Main Results}

In this section, we establish the existence and uniqueness of positive solutions results for the problems (1).

Theorem 4. Assume that $f_{1} \in C[[0,1] \times[0, \infty) \times[0, \infty)$, $[0, \infty)]$ and $f_{2} \in C[[0,1] \times[0, \infty),[0, \infty)]$ are continuous. A function $x \in P$ is a solution of the BVP (1) if and only if it is a solution of the integral equation

$$
\begin{aligned}
x(t)= & \int_{0}^{1} G(t, s)\left[f_{1}(s, x(s), x(s))+f_{2}(s, x(s))\right] d s \\
& +\lambda \frac{t^{\alpha-1}}{1-\sum_{i=1}^{m-2} \alpha_{i} \xi_{i}^{\alpha-1}} .
\end{aligned}
$$

Proof. The proof of this result is quite similar to that given earlier in Lemma 3 and omitted.

Define operators $T$ and $S$ as follows:

$$
\begin{aligned}
T(x, y)(t)= & \int_{0}^{1} G(t, s) f_{1}(s, x(s), y(s)) d s, \\
(S x)(t)= & \int_{0}^{1} G(t, s) f_{2}(s, x(s)) d s \\
& +\lambda \frac{t^{\alpha-1}}{1-\sum_{i=1}^{m-2} \alpha_{i} \xi_{i}^{\alpha-1}} .
\end{aligned}
$$

Theorem 5. Assume that

$\left(\mathrm{H}_{1}\right) f_{1}(t, x, y) \in C[[0,1] \times[0,+\infty) \times[0,+\infty),[0,+\infty)]$, $f_{2}(t, x) \in C[[0,1] \times[0,+\infty) \rightarrow[0,+\infty)], f_{1}$ and $f_{2}$ increasing in $x \in[0,+\infty), f$ decreasing in $y \in$ $[0,+\infty)$, and there exists a constant $\delta_{0}>0$ such that $f_{1}(t, x, y) \geq \delta_{0} f_{2}(t, x)+\delta_{0}^{2}\left(1 /\left(1-\sum_{i=1}^{m-2} \alpha_{i} \xi_{i}^{\alpha-1}\right)\right) ;$

$\left(\mathrm{H}_{2}\right)$ for all $\mu \in(0,1), t \in[0,1], x, y \in[0,+\infty)$, there exists a constant $\xi \in(0,1)$ such that $f_{2}(t, \mu x) \geq \mu f_{2}(t, x)$ and $f_{1}\left(t, \mu x, \mu^{-1} y\right) \geq \mu^{\xi} f_{1}(t, x, y)$.
Then, for any $0<\lambda \leq \delta_{0}, B V P(1)$ has a unique positive solution $x^{*}$ in $P_{h}$, where $h(t)=t^{\alpha-1}$, and for any $x_{0}, y_{0} \in P_{h}$, construct successively the sequences

$$
\begin{aligned}
& x_{n+1}(t) \\
& =\int_{0}^{1} G(t, s)\left[f_{1}\left(s, x_{n}(s), y_{n}(s)\right)+f_{2}\left(s, x_{n}(s)\right)\right] d s \\
& +\lambda \frac{t^{\alpha-1}}{1-\sum_{i=1}^{m-2} \alpha_{i} \xi_{i}^{\alpha-1}}, \quad n=0,1,2, \ldots, \\
& y_{n+1}(t) \\
& =\int_{0}^{1} G(t, s)\left[f_{1}\left(s, y_{n}(s), x_{n}(s)\right)+f_{2}\left(s, y_{n}(s)\right)\right] d s \\
& +\lambda \frac{t^{\alpha-1}}{1-\sum_{i=1}^{m-2} \alpha_{i} \xi_{i}^{\alpha-1}}, \quad n=0,1,2, \ldots,
\end{aligned}
$$

Proof. According to Theorem 4 and the definition of operators $T$ and $S$, it is straightforward to show that $x$ is the solution of BVP (1) if and only if $x$ solves the operator equation $x=$ $T(x, x)+S x$. We only need to investigate the solution of the above operator equation.

Now, we will show that $T: P \times P \rightarrow P$ and $S: P \rightarrow$ $P$ satisfy all the assumptions of Lemma 1 . To begin with, we prove that $A$ is a mixed monotones operator. In fact, for $x_{i}$, $y_{i} \in P, i=1,2$ with $x_{1} \geq x_{2}, y_{1} \leq y_{2}$, we know that $x_{1}(t) \geq$ $x_{2}(t)$ and $y_{1}(t) \leq y_{2}(t)$ for all $t \in[0,1]$. According to $\left(\mathrm{H}_{1}\right)$ and Lemma 3 , we get

$$
f_{1}\left(s, x_{1}(s), y_{1}(s)\right) \geq f_{1}\left(s, x_{2}(s), y_{2}(s)\right),
$$

since $G(t, s)>0$, that is, $T\left(x_{1}, y_{1}\right) \geq T\left(x_{2}, y_{2}\right)$. Furthermore, an argument similar to the one used in $T$ shows that $S$ is increasing. Next we prove that $T$ satisfies (8). For any $\mu, \xi \in$ $(0,1)$ and $x, y \in P$, from

$$
f_{1}\left(s, \mu x(s), \mu^{-1} y(s)\right) d s \geq \mu^{\xi} f_{1}(s, x(s), y(s))
$$

we have $T\left(\mu x, \mu^{-1} y\right) \geq \mu^{\xi} T(x, y)$ for $\mu, \xi \in(0,1), x, y \in P$. Hence (8) holds. Also, for any $\mu \in(0,1), x \in P$, taking $\left(\mathrm{H}_{2}\right)$ into consideration, we have

$$
\begin{aligned}
S(\mu x)(t)= & \int_{0}^{1} G(t, s) f_{2}(s, \mu x(s)) d s \\
& +\lambda \frac{t^{\alpha-1}}{1-\sum_{i=1}^{m-2} \alpha_{i} \xi_{i}^{\alpha-1}} \\
\geq & \mu \int_{0}^{1} G(t, s) f_{2}(s, x(s)) d s \\
& +\mu \lambda \frac{t^{\alpha-1}}{1-\sum_{i=1}^{m-2} \alpha_{i} \xi_{i}^{\alpha-1}}=\mu(S x)(t) .
\end{aligned}
$$

Thus, $S(\mu x) \geq \mu S(x)$ for any $\mu \in(0,1), x \in P$. Hence the operator $S$ is subhomogeneous. Now, we are in the position 
to show that $T(h, h) \in P_{h}$ and $S h \in P_{h}$. It follows from $\left(\mathrm{H}_{1}\right)$, $\left(\mathrm{H}_{2}\right)$ and Lemma 3 that, for any $t \in[0,1]$,

$$
\begin{aligned}
& T(h, h)(t)=\int_{0}^{1} G(t, s) f_{1}(s, h(s), h(s)) d s \leq h(t) \\
& \cdot \int_{0}^{1}\left[1+\frac{1}{1-\sum_{i=1}^{m-2} \alpha_{i} \xi_{i}^{\alpha-1}} \sum_{i=1}^{m-2} \alpha_{i} H\left(\xi_{i}, s\right)\right] \\
& \cdot f_{1}(s, 0,1) d s=a_{2} h(t),
\end{aligned}
$$

where

$$
\begin{aligned}
a_{2} & :=\int_{0}^{1}\left[1+\frac{1}{1-\sum_{i=1}^{m-2} \alpha_{i} \xi_{i}^{\alpha-1}} \sum_{i=1}^{m-2} \alpha_{i} H\left(\xi_{i}, s\right)\right] \\
& \cdot f_{1}(s, 0,1) d s, \\
T & (h, h)(t)=\int_{0}^{1} G(t, s) f_{1}(s, h(s), h(s)) d s \\
& \geq \frac{h(t)}{1-\sum_{i=1}^{m-2} \alpha_{i} \xi_{i}^{\alpha-1}} \int_{0}^{1} \sum_{i=1}^{m-2} \alpha_{i} H\left(\xi_{i}, s\right) f_{1}(s, 1,0) d s \\
& =a_{1} h(t),
\end{aligned}
$$

where

$$
a_{1}:=\int_{0}^{1} \frac{1}{1-\sum_{i=1}^{m-2} \alpha_{i} \xi_{i}^{\alpha-1}} \sum_{i=1}^{m-2} \alpha_{i} H\left(\xi_{i}, s\right) f_{1}(s, 1,0) d s,
$$

Thanks to $\left(\mathrm{H}_{1}\right),\left(\mathrm{H}_{2}\right)$ and $\left(\mathrm{H}_{3}\right)$, we get

$$
\begin{aligned}
f_{1}(s, 1,0) & \geq f_{1}(s, 0,1) \\
& \geq \delta_{0} f_{2}(s, 0)+\delta_{0}^{2} \frac{1}{1-\sum_{i=1}^{m-2} \alpha_{i} \xi_{i}^{\alpha-1}}>0 .
\end{aligned}
$$

So

$$
\begin{aligned}
\int_{0}^{1} f_{1}(s, 1,0) d s \geq & \delta_{0} \int_{0}^{1} f_{2}(s, 0) d s \\
& +\delta_{0}^{2} \frac{1}{1-\sum_{i=1}^{m-2} \alpha_{i} \xi_{i}^{\alpha-1}}>0,
\end{aligned}
$$

and, in consequence, $a_{1}>0$ and $a_{2}>0$. Thus, for $t \in[0,1]$, inequality $a_{1} h(t) \leq T(h, h)(t) \leq a_{2} h(t)$ holds, and then we get $T(h, h) \in P_{h}$. An argument similar to the one used in $T$ shows that

$$
\begin{gathered}
S h(t)=\int_{0}^{1} G(t, s) f_{2}(s, h(s)) d s+\lambda \frac{t^{\alpha-1}}{1-\sum_{i=1}^{m-2} \alpha_{i} \xi_{i}^{\alpha-1}} \\
\leq h(t)\left\{\int_{0}^{1}\left[1+\frac{1}{1-\sum_{i=1}^{m-2} \alpha_{i} \xi_{i}^{\alpha-1}} \sum_{i=1}^{m-2} \alpha_{i} H\left(\xi_{i}, s\right)\right]\right. \\
\left.\cdot f_{2}(s, 0) d s+\frac{\delta_{0}}{1-\sum_{i=1}^{m-2} \alpha_{i} \xi_{i}^{\alpha-1}}\right\}=b_{2} h(t),
\end{gathered}
$$

where

$$
\begin{aligned}
b_{2}:= & \int_{0}^{1}\left[1+\frac{1}{1-\sum_{i=1}^{m-2} \alpha_{i} \xi_{i}^{\alpha-1}} \sum_{i=1}^{m-2} \alpha_{i} H\left(\xi_{i}, s\right)\right] \\
& \cdot f_{2}(s, 0) d s+\frac{\delta_{0}}{1-\sum_{i=1}^{m-2} \alpha_{i} \xi_{i}^{\alpha-1}}, \\
S h(t) & =\int_{0}^{1} G(t, s) f_{2}(s, h(s)) d s+\lambda \frac{t^{\alpha-1}}{1-\sum_{i=1}^{m-2} \alpha_{i} \xi_{i}^{\alpha-1}} \\
& \geq h(t)\left\{\int_{0}^{1} \frac{1}{1-\sum_{i=1}^{m-2} \alpha_{i} \xi_{i}^{\alpha-1}}\right. \\
& \left.\cdot \sum_{i=1}^{m-2} \alpha_{i} H\left(\xi_{i}, s\right) f_{2}(s, 1) d s\right\}=b_{1} h(t),
\end{aligned}
$$

where

$$
b_{1}:=\int_{0}^{1} \frac{1}{1-\sum_{i=1}^{m-2} \alpha_{i} \xi_{i}^{\alpha-1}} \sum_{i=1}^{m-2} \alpha_{i} H\left(\xi_{i}, s\right) f_{2}(s, 1) d s .
$$

Obviously, $b_{1}>0$ and $b_{2}>0$; thus we are led to the conclusion that $b_{1} h(t) \leq S h(t) \leq b_{2} h(t)$ with $t \in[0,1]$, i.e., Sh $\in P_{h}$. Hence condition (i) of Lemma 1 is proved. It remains to show that condition (ii) of Lemma 1 is satisfied. For any $x, y \in P, t \in$ $[0,1]$, from $\left(\mathrm{H}_{3}\right)$ we have

$$
\begin{aligned}
& T(x, y)(t)=\int_{0}^{1} G(t, s) f_{1}(s, x(s), y(s)) d s \\
& \quad \geq \delta_{0} \int_{0}^{1} G(t, s)\left[f_{2}(s, x(s))+\frac{\delta_{0}}{1-\sum_{i=1}^{m-2} \alpha_{i} \xi_{i}^{\alpha-1}}\right] d s \\
& \quad \geq \delta_{0}(S x)(t) .
\end{aligned}
$$

That is, for any $x, y \in P$, inequality $T(x, y) \geq \delta_{0} S x$ holds. Applying Lemma 2, we can get the conclusion of Theorem 5.

As a special case of Theorem 5, we obtain the following results.

\section{Corollary 6. Assume that}

$\left(\mathrm{H}_{1}\right)^{\prime} f(t, x, y) \in C[[0,1] \times[0,+\infty) \times[0,+\infty),[0,+\infty)]$ is increasing in $x \in[0,+\infty)$, decreasing in $y \in[0,+\infty)$ and $f(t, 0,1) \neq \equiv$;

$\left(\mathrm{H}_{2}\right)^{\prime}$ for all $t \in[0,1], \mu \in(0,1), x, y \in[0,+\infty)$, there exists a constant $\xi \in(0,1)$ such that $f\left(t, \mu x, \mu^{-1} y\right) \geq$ $\mu^{\xi} f(t, x, y)$.

Then, the problem

$$
\begin{aligned}
& -D_{0+}^{\alpha} x(t)=f(t, x(t), x(t)), \\
& 0<t<1, n-1<\alpha \leq n, n \geq 2,
\end{aligned}
$$




$$
\begin{aligned}
& x(0)=x^{(\prime)}(0)=\cdots=x^{(k)}(0)=0, \\
& \quad 0 \leq k \leq n-2, \\
& x(1)=\sum_{i=1}^{m-2} \alpha_{i} x\left(\xi_{i}\right)+\lambda, \quad m \geq 3
\end{aligned}
$$

has a unique positive solution $x^{*}$ in $P_{h}$, where $h(t)=t^{\alpha-1}$ and, for any $x_{0}, y_{0} \in P_{h}$, constructing successively the sequences

$$
\begin{aligned}
x_{n+1}(t)= & \int_{0}^{1} G(t, s) f\left(s, x_{n}(s), y_{n}(s)\right) d s \\
& +\lambda \frac{t^{\alpha-1}}{1-\sum_{i=1}^{m-2} \alpha_{i} \xi_{i}^{\alpha-1}}, \quad n=0,1,2, \ldots, \\
y_{n+1}(t)= & \int_{0}^{1} G(t, s) f\left(s, y_{n}(s), x_{n}(s)\right) d s \\
& +\lambda \frac{t^{\alpha-1}}{1-\sum_{i=1}^{m-2} \alpha_{i} \xi_{i}^{\alpha-1}}, \quad n=0,1,2, \ldots,
\end{aligned}
$$

we have $\left\|x_{n}-x^{*}\right\| \rightarrow 0$ and $\left\|y_{n}-x^{*}\right\| \rightarrow 0$ as $n \rightarrow \infty$.

Proof. Letting $g \equiv 0$ in Theorem 5, by suitable modification to the proof of Theorem 5, we can prove the desired conclusion.

If $T: P \times P \rightarrow P$ is a mixed monotone operator and satisfies

$$
T\left(t x, t^{-1} y\right) \geq t T(x, y), \quad \forall t \in(0,1), x, y \in P .
$$

$S: P \rightarrow P$ is an increasing $\alpha$-concave operator. We can also obtain the following result.

Theorem 7. Assume that

$\left(\mathrm{H}_{3}\right) f_{1}(t, x, y) \in C[[0,1] \times[0,+\infty) \times[0,+\infty),[0,+\infty)]$, $f_{2}(t, x) \in C[[0,1] \times[0,+\infty) \rightarrow[0,+\infty)], f_{1}$ and $f_{2}$ increasing in $x \in[0,+\infty), f$ decreasing in $y \in$ $[0,+\infty)$, and there exists a constant $\delta_{0}>0$ such that $f_{1}(t, x, y) \leq \delta_{0} f_{2}(t, x)+\delta_{0}^{2}\left(1 /\left(1-\sum_{i=1}^{m-2} \alpha_{i} \xi_{i}^{\alpha-1}\right)\right) ;$

$\left(\mathrm{H}_{4}\right)$ for all $\in[0,1], \mu \in(0,1), x, y \in[0,+\infty)$, there exists a constant $\xi \in(0,1)$ such that $f_{2}(t, \mu x) \geq \mu^{\xi} f_{2}(t, x)$ and $f_{1}\left(t, \mu x, \mu^{-1} y\right) \geq \mu f_{1}(t, x, y)$.

Then, BVP (1) has a unique positive solution $x^{*}$ in $P_{h}$, where $h(t)=t^{\alpha-1}$ and, for any $x_{0}, y_{0} \in P_{h}$, constructing successively the sequences

$$
\begin{aligned}
& x_{n+1}(t) \\
& =\int_{0}^{1} G(t, s)\left[f_{1}\left(s, x_{n}(s), y_{n}(s)\right)+f_{2}\left(s, x_{n}(s)\right)\right] d s \\
& \quad+\lambda \frac{t^{\alpha-1}}{1-\sum_{i=1}^{m-2} \alpha_{i} \xi_{i}^{\alpha-1}}, \quad n=0,1,2, \ldots,
\end{aligned}
$$

$$
\begin{aligned}
& y_{n+1}(t) \\
& =\int_{0}^{1} G(t, s)\left[f_{1}\left(s, y_{n}(s), x_{n}(s)\right)+f_{2}\left(s, y_{n}(s)\right)\right] d s \\
& \quad+\lambda \frac{t^{\alpha-1}}{1-\sum_{i=1}^{m-2} \alpha_{i} \xi_{i}^{\alpha-1}}, \quad n=0,1,2, \ldots,
\end{aligned}
$$

we have $\left\|x_{n}-x^{*}\right\| \rightarrow 0$ and $\left\|y_{n}-x^{*}\right\| \rightarrow 0$ as $n \rightarrow \infty$.

Proof. In the light of [26, Theorem 2.4], a similar argument used in Theorem 5 . shows that the desire result. We omit it.

\section{Example}

In this section, we give one example to illustrate our results.

Example 1. Consider the BVP (1). Letting $\alpha=5 / 2, \alpha_{1}=1 / 2$, $\alpha_{2}=1 / 4, \xi_{1}=1 / 16, \xi_{2}=1 / 4, f_{1}(t, x, y)=x^{1 / 2}+y^{-1 / 2}+$ $t^{2}+8 / 123$ and $f_{2}(t, x)=x^{1 / 2}+t^{3}$. A simple calculation gives $\sum_{i=1}^{m-2} \alpha_{i} \xi_{i}^{\alpha-1}=5 / 128<1$. It is not difficult to verify that $\left(\mathrm{H}_{1}\right)$ and $\left(\mathrm{H}_{2}\right)$ are satisfied. Taking $\delta_{0}=1 / 4>0$, we have

$$
\begin{aligned}
f_{1}(t, x, y) & =x^{1 / 2}+y^{-1 / 2}+t^{2}+\frac{8}{123} \\
& \geq x^{1 / 2}+t^{3}+\frac{8}{123} \\
& \geq \delta_{0}\left(x^{1 / 2}+t^{3}+\delta_{0} \frac{128}{123}\right) \\
& =\delta_{0}\left[f_{2}(t, x)+\delta_{0} \frac{1}{1-\sum_{i=1}^{m-2} \alpha_{i} \xi_{i}^{\alpha-1}}\right] .
\end{aligned}
$$

Thus, $\left(\mathrm{H}_{3}\right)$ is satisfied. Moreover, for any $\mu \in(0,1), t \in[0,1]$, $x \in[0, \infty), y \in[0, \infty)$, we have

$$
\begin{aligned}
f_{2}(t, \mu x) & =\mu^{1 / 2} x^{1 / 2}+t^{3} \geq \mu^{1 / 2}\left(x^{1 / 2}+t^{3}\right) \\
& \geq \mu f_{2}(t, x), \\
f_{1}\left(t, \mu x, \mu^{-1} y\right) & =\mu^{1 / 2} x^{1 / 2}+\mu^{1 / 2} y^{-1 / 2}+t^{2}+\frac{8}{123} \\
& \geq \mu^{1 / 2}\left(x^{1 / 2}+y^{-1 / 2}+t^{2}+\frac{8}{123}\right) \\
& =\mu^{\gamma} f_{1}(t, x, y),
\end{aligned}
$$

where $\gamma=1 / 2$. We conclude that condition $\left(\mathrm{H}_{4}\right)$ is satisfied. Therefore, Theorem 5 ensures that the BVP (1) has a unique positive solution in $P_{h}$ with $h(t)=t^{3 / 2}$.

\section{Data Availability}

No data were used to support this study. 


\section{Conflicts of Interest}

The authors declare that there are no conflicts of interest regarding the publication of this paper.

\section{Authors' Contributions}

All authors typed, read, and approved the final manuscript.

\section{Acknowledgments}

This project is supported by Shandong Provincial Natural Science Foundation (Grant no. ZR2017LA002), Weifang Science and Technology Development Projects (Grant no. 2017GX025), and Doctoral Research Foundation of Weifang University (Grant no. 2017BS02).

\section{References}

[1] V. Lakshmikantham and A. S. Vatsala, "General uniqueness and monotone iterative technique for fractional differential equations," Applied Mathematics Letters, vol. 21, no. 8, pp. 828834, 2008.

[2] W. Rudin, Functional Analysis. International Series in Pure and Applied Mathematics, McGraw-Hill, New York, NY, USA, 2nd edition, 1991.

[3] S. G. Samko, A. A. Kilbas, and O. I. Marichev, Fractional Integrals and Derivatives, Theory and Applications, Gordon and Breach, Yverdon, Switzerland, 1993.

[4] R. P. Agarwal, D. O’Regan, and S. Stanek, "Positive solutions for Dirichlet problems of singular nonlinear fractional differential equations," Journal of Mathematical Analysis and Applications, vol. 371, no. 1, pp. 57-68, 2010.

[5] X. Xu, D. Jiang, W. Hu, D. O’Regan, and R. P. Agarwal, "Positive properties of Green's function for three-point boundary value problems of nonlinear fractional differential equations and its applications," Applicable Analysis: An International Journal, vol. 91, no. 2, pp. 323-343, 2012.

[6] J. R. Webb and M. Zima, "Multiple positive solutions of resonant and non-resonant nonlocal boundary value problems," Nonlinear Analysis, Theory, Methods \& Applications, vol. 71, no. 3-4, pp. 1369-1378, 2009.

[7] K. Zhang and X.-J. Xie, "Existence of sign-changing solutions for some asymptotically linear three-point boundary value problems," Nonlinear Analysis, Theory, Methods \& Applications, vol. 70, no. 7, pp. 2796-2805, 2009.

[8] K. M. Zhang, "On a sign-changing solution for some fractional differential equations," Boundary Value Problems, vol. 59, pp. 112, 2017.

[9] K. Zhang, "Nontrivial solutions of fourth-order singular boundary value problems with sign-changing nonlinear terms," Topological Methods in Nonlinear Analysis, vol. 40, no. 1, pp. 5370, 2012.

[10] X. Zhang, L. Liu, Y. Wu, and B. Wiwatanapataphee, “The spectral analysis for a singular fractional differential equation with a signed measure," Applied Mathematics and Computation, vol. 257, pp. 252-263, 2015.

[11] X. Zhang, L. Liu, and Y. Wu, "The entire large solutions for a quasilinear Schrodinger elliptic equation by the dual approach," Applied Mathematics Letters, vol. 55, pp. 1-9, 2016.
[12] J. Tan and C. Cheng, "Fractional boundary value problems with Riemann-Liouville fractional derivatives," Advances in Difference Equations, vol. 80, pp. 1-14, 2015.

[13] X. Zhang, L. Liu, and Y. Wu, "The uniqueness of positive solution for a fractional order model of turbulent flow in a porous medium," Applied Mathematics Letters, vol. 37, pp. 2633, 2014.

[14] L. Liu, F. Sun, X. Zhang, and Y. Wu, "Bifurcation analysis for a singular differential system with two parameters via to topological degree theory," Lithuanian Association of Nonlinear Analysts. Nonlinear Analysis: Modelling and Control, vol. 22, no. 1, pp. 31-50, 2017.

[15] L. Liu, H. Li, C. Liu, and Y. Wu, "Existence and uniqueness of positive solutions for singular fractional differential systems with coupled integral boundary conditions," The Journal of Nonlinear Science and its Applications, vol. 10, no. 1, pp. 243-262, 2017.

[16] X. Lin and Z. Zhao, "Existence and uniqueness of symmetric positive solutions of $2 \mathrm{n}$-order nonlinear singular boundary value problems," Applied Mathematics Letters, vol. 26, no. 7, pp. 692-698, 2013.

[17] Y. Wang and L. Liu, "Positive solutions for a class of fractional 3-point boundary value problems at resonance," Advances in Difference Equations, Paper No. 7, 13 pages, 2017.

[18] J. Jiang, L. Liu, and Y. Wu, "Positive solutions to nonlinear fractional differential equations involving Stieltjes integrals conditions," Journal of Nonlinear Sciences and Applications. JNSA, vol. 10, no. 10, pp. 5360-5372, 2017.

[19] Y. Wang and J. Jiang, "Existence and nonexistence of positive solutions for the fractional coupled system involving generalized p-Laplacian," Advances in Difference Equations, Paper No. 337, 19 pages, 2017.

[20] J. J. Tan and Cheng. C. Z., "Postive solution of fractional differential equation nonlocal boundary value problems," Advances in Differential Equations, vol. 256, pp. 1-14, 2015.

[21] L. Liu, X. Zhang, J. Jiang, and Y. Wu, "The unique solution of a class of sum mixed monotone operator equations and its application to fractional boundary value problems," Journal of Nonlinear Sciences and Applications. JNSA, vol. 9, no. 5, pp. 2943-2958, 2016.

[22] L. Liu, D. Kong, and Y. Wu, "The best approximation theorems and variational inequalities for discontinuous mappings in Banach spaces," Science China Mathematics, vol. 58, no. 12, pp. 2581-2592, 2015.

[23] X. Zhang, L. Liu, Y. Wu, and L. Caccetta, "Entire large solutions for a class of Schrodinger systems with a nonlinear random operator," Journal of Mathematical Analysis and Applications, vol. 423, no. 2, pp. 1650-1659, 2015.

[24] A. M. El-Sayed and E. O. Bin-Taher, "Positive solutions for a nonlocal multi-point boundary-value problem of fractional and second order," Electronic Journal of Differential Equations, vol. 64, pp. 1-8, 2013.

[25] S. Zhang, "Positive solutions to singular boundary value problem for nonlinear fractional differential equation," Computers \& Mathematics with Applications. An International Journal, vol. 59, no. 3, pp. 1300-1309, 2010.

[26] C. Zhai and M. Hao, "Fixed point theorems for mixed monotone operators with perturbation and applications to fractional differential equation boundary value problems," Nonlinear Analysis, Theory, Methods \& Applications, vol. 75, no. 4, pp. 2542-2551, 2012. 


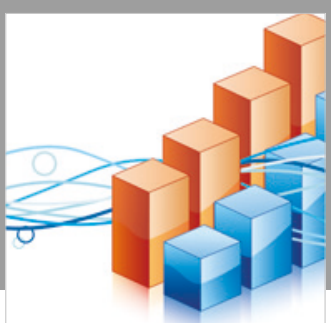

Advances in

Operations Research

\section{-n-m}
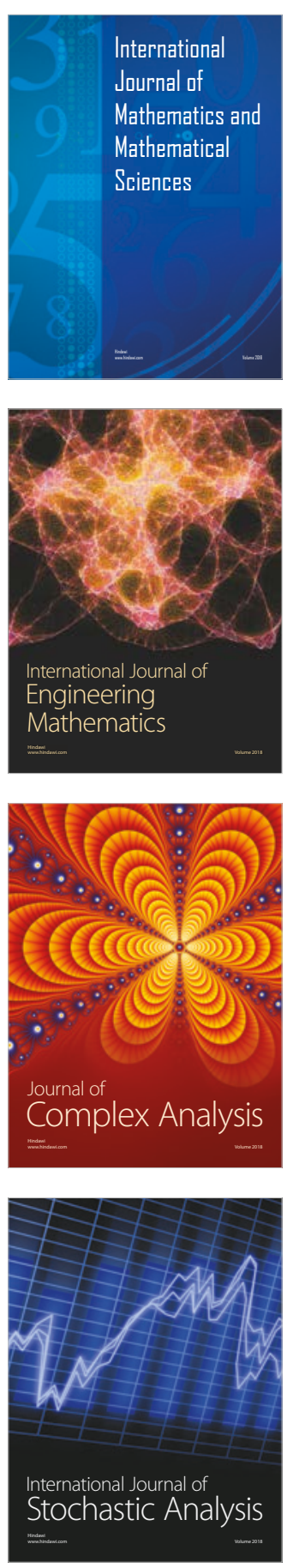
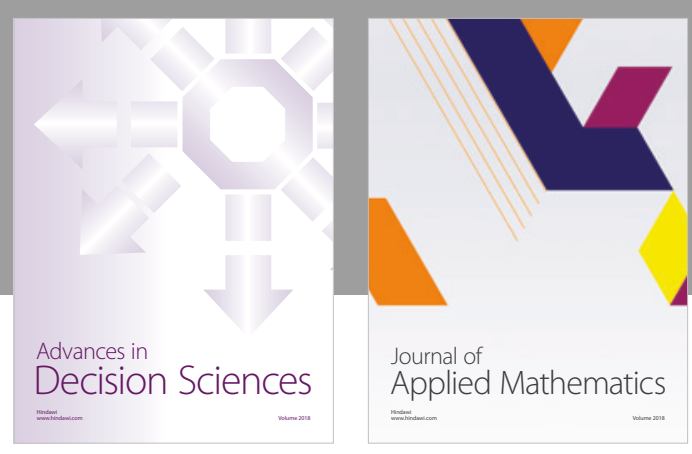

Journal of

Applied Mathematics
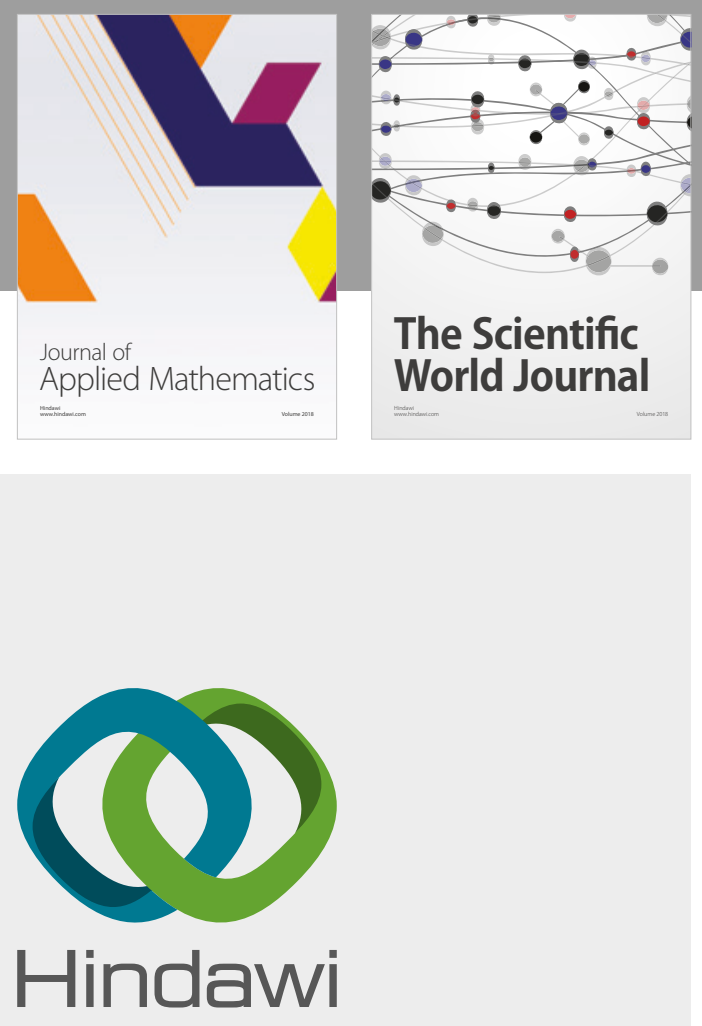

Submit your manuscripts at

www.hindawi.com

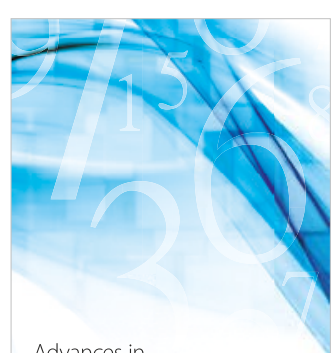

Advances in
Numerical Analysis
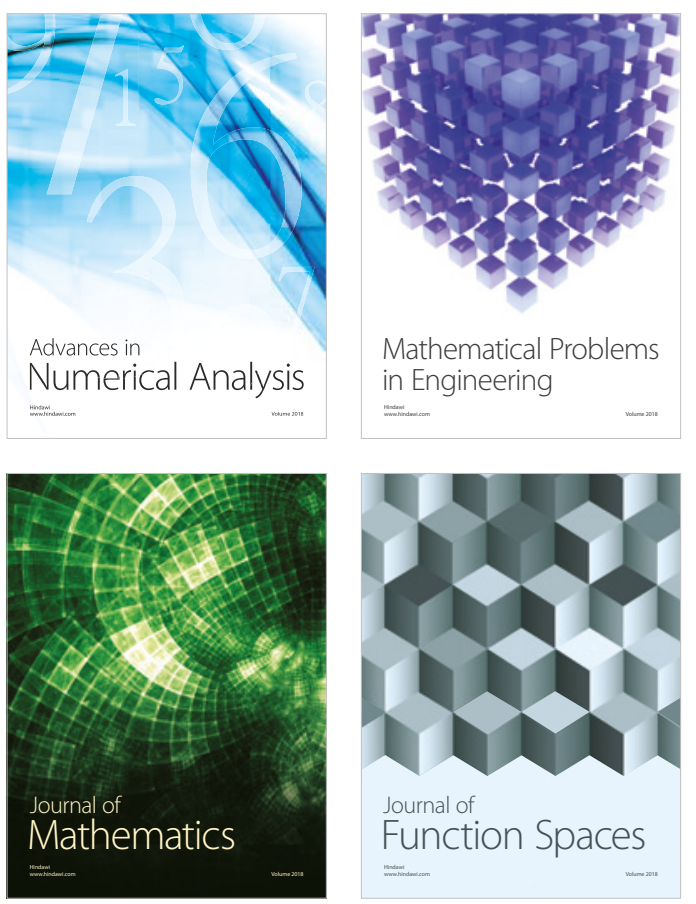

Mathematical Problems in Engineering

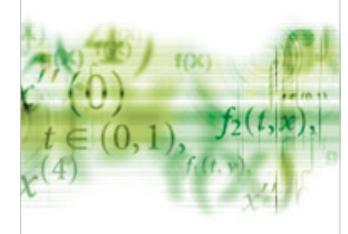

International Journal of

Differential Equations

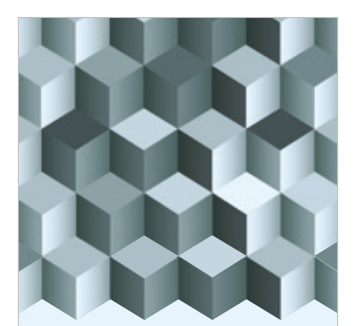

Journal of

Function Spaces

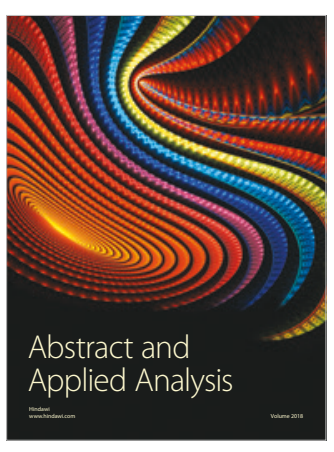

The Scientific

World Journal

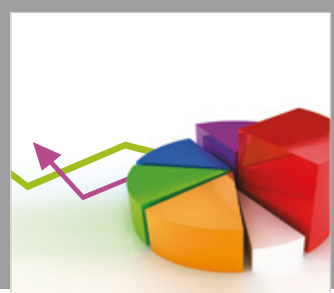

Journal of

Probability and Statistics
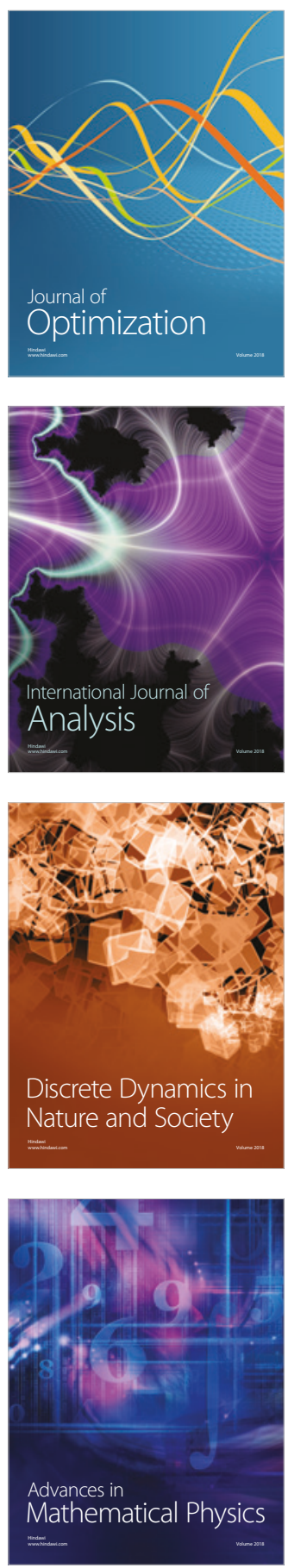